\title{
Megaprepucio congénito: diagnóstico y manejo terapéutico
}

\author{
Delgado Oliva FJ, Domínguez Hinarejos C, Serrano Durbá S, Estornell Moragues F, \\ Martínez Verduch M, García Ibarra F.
}

Unidad de Urología Pediátrica. Hospital Universitario La Fe. Valencia.

Actas Urol Esp. 2006;30(10):1038-1042

\section{RESUMEN}

\section{MEGAPREPUCIO CONGÉNITO: DIAGNÓSTICO Y MANEJO TERAPÉUTICO}

Objetivo: Analizar la presentación clínica, diagnóstico y manejo terapéutico del megaprepucio congénito (MC).

Material y métodos: Estudio retrospectivo de los 4 casos de MC diagnosticados y tratados en nuestro Servicio desde octubre 1997 hasta abril de 2005, describiendo el diagnóstico y la técnica quirúrgica empleada en todos ellos.

Conclusiones: El MC es una condición infrecuente, que puede confundirse con otras patologías, que es necesario reconocer y tratar correctamente por parte del Urólogo.

Palabras Clave: Megaprepucio congénito. Diagnóstico. Tratamiento quirúrgico.

\section{ABSTRACT}

CONGENITAL MEGAPREPUCE: DIAGNOSIS AND TERAPEUTIC MANAGEMENT

Objetive: To analize the clinical presentation, diagnosis and surgical management of congenital megaprepuce $(\mathrm{CM})$.

Material and methods: We have made a retrospective study of four cases of CM diagnosed and treated in our centre between october 1997 and april 2005. We studied the diagnosis and surgical treatment.

Conclusions: CM is an infrecuent condition that can be confused with others pathologies. It's necessary to diagnose and treat it correctly.

Keywords: Congenital megaprepuce. Diagnosis. Surgical treatment.

$\mathrm{E}^{\prime}$ término megaprepucio congénito (MC) fue descrito por primera vez en 1994 por O`Brien ${ }^{1}$. Se trata de un prepucio que, al nacimiento, se caracteriza por presentar una mucosa muy extensa y redundante acompañada de piel prepucial escasa y engrosada, que cubre totalmente el glande sobre un pene de tamaño y aspecto normales $^{4}$. El megaprepucio congénito o primario no debe ser confundido con el megaprepucio secundario, que tiene su origen en la constricción del prepucio por la ropa interior del niño sobre un prepucio ya redundante, también puede ser secundario a fimosis ${ }^{2,4,8}$.
El MC suele presentarse clínicamente por una masa de contenido urinario durante la micción en la porción ventral del pene que, generalmente, se extiende hacia el escroto ${ }^{3}$. El diagnóstico de esta patología es clínico, mediante la exploración física del niño, sin precisar exploraciones complementarias adicionales. Se trata de una patología poco frecuente que es importante conocer, no sólo por las implicaciones estéticas que conlleva, sino también por la eventual afectación del tractor urinario superior condicionada por la dificultad miccional secundaria al estrechamiento distal del pene por el prepucio redundante ${ }^{3,4}$. 


\section{MATERIAL Y MÉTODOS}

Hemos realizado un estudio retrospectivo de los 4 casos de MC diagnosticados y tratados en nuestro Centro en el periodo comprendido de octubre de 1997 a junio 2005, describiendo la técnica de corrección quirúrgica empleada en todos ellos. La cirugía se llevó a cabo antes del año de vida en los cuatro casos.

En las Figuras la y $1 \mathrm{~b}$ podemos apreciar la tumefacción penoescrotal típica del MC y la necesidad de realizar la descompresión manual de la misma para evacuar su contenido urinario, así como el defecto de piel prepucial ventral.

La cirugía comienza con la realización de una incisión dorsal en la piel del prepucio, que expone una mucosa prepucial excesivamente redundante (Fig. 2a).

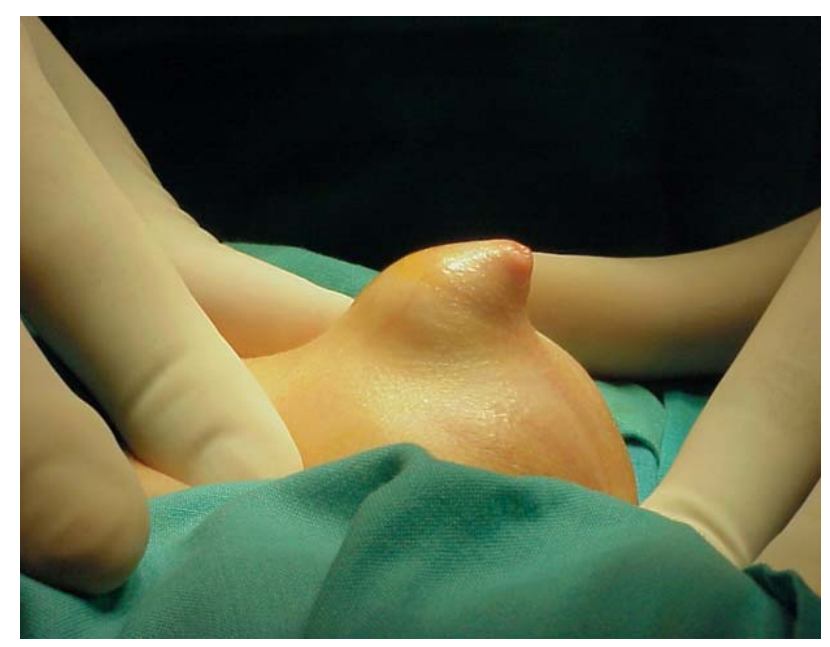

\section{FIGURA 1a}

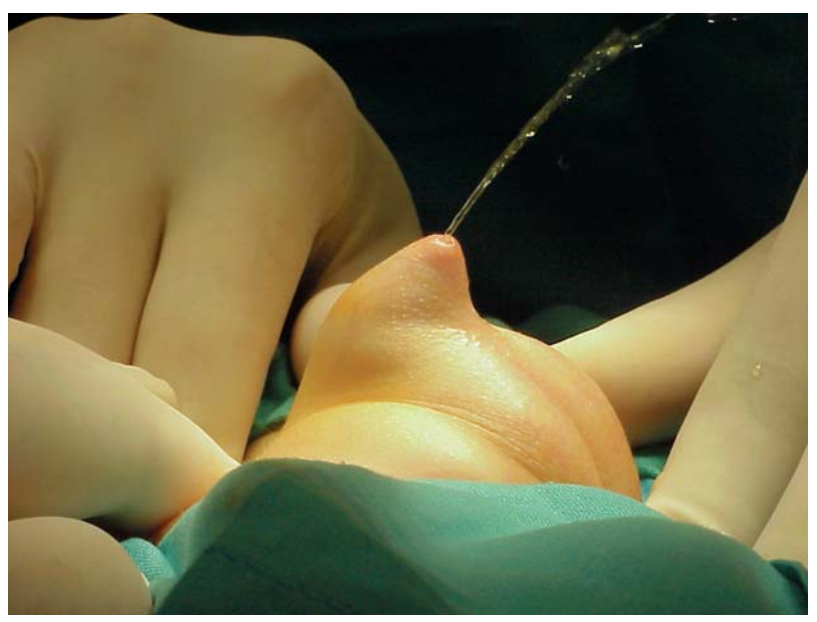

FIGURA $1 b$

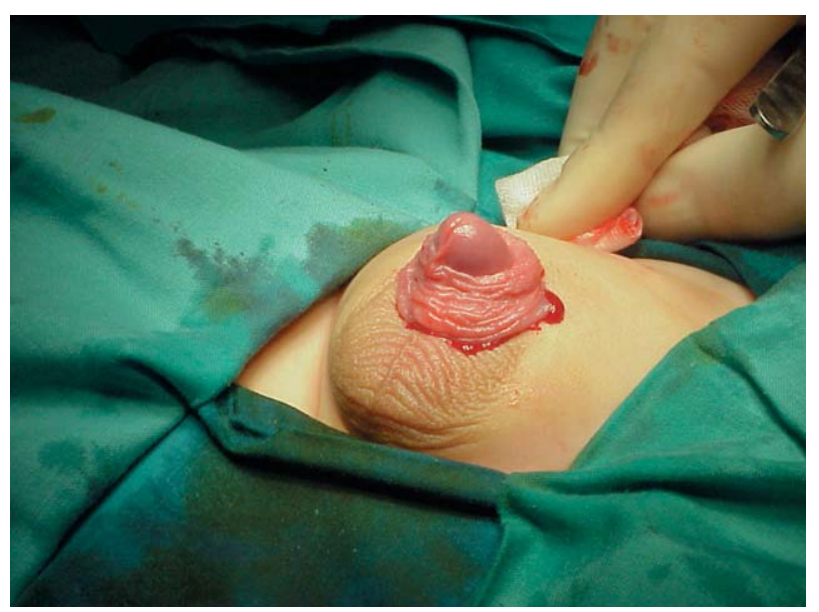

FIGURA $2 a$

Realizamos una extensión de toda esta mucosa para saber la longitud de la misma que podemos utilizar para la reconstrucción, procediendo posteriormente a la frenuloplastia reglada con sutura reabsorbible de 5/0 (Figs. 2b y 2c).

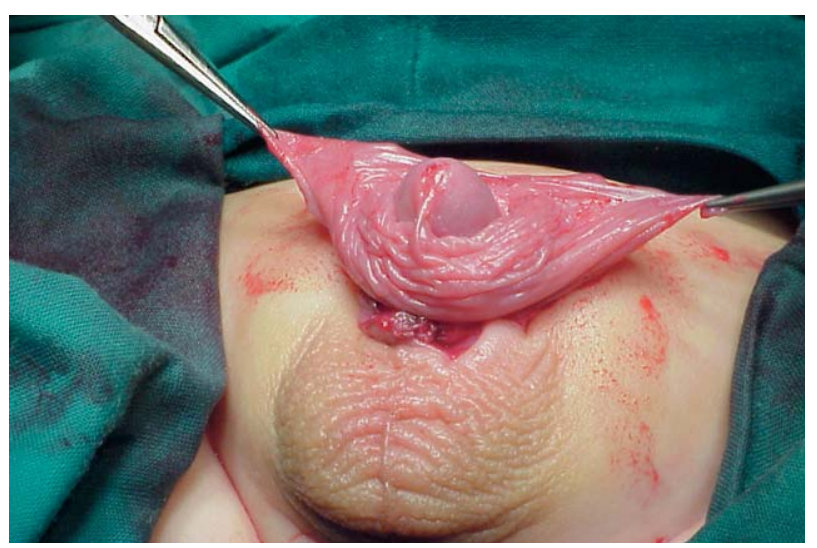

FIGURA 2b

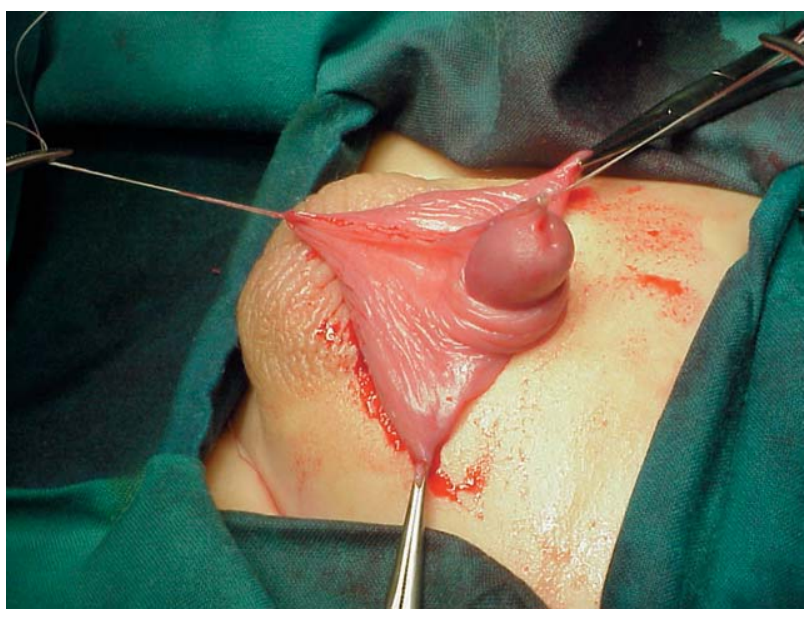

FIGURA 2c 
Marcamos con un rotulador la zona dorsal de la mucosa prepucial, incindiendo la misma y mobilizándola hacia la cara ventral del pene (Figs. 3a y 3b).

Seguidamente procedemos a la resección de la mucosa redundante y a la sutura de la misma a la reducida piel ventral que suele encontrarse en estos casos (Fig. 3c). Finalizamos la intervención

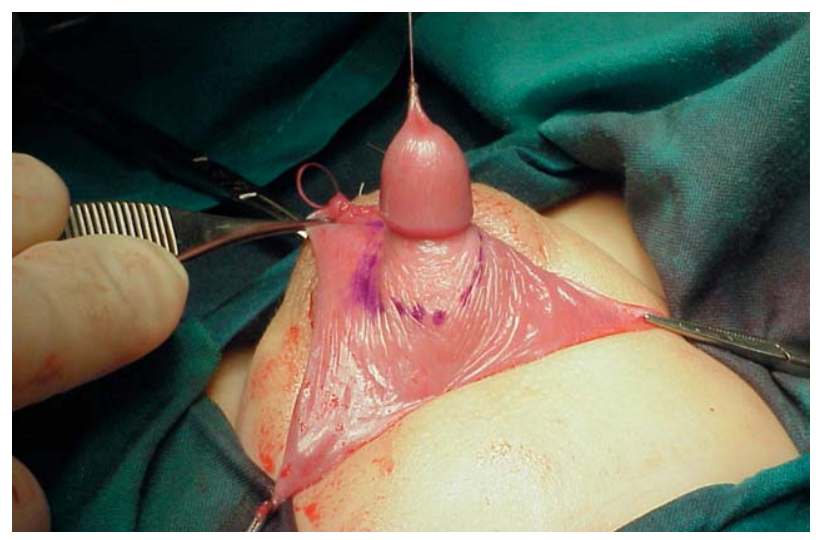

FIGURA $3 a$

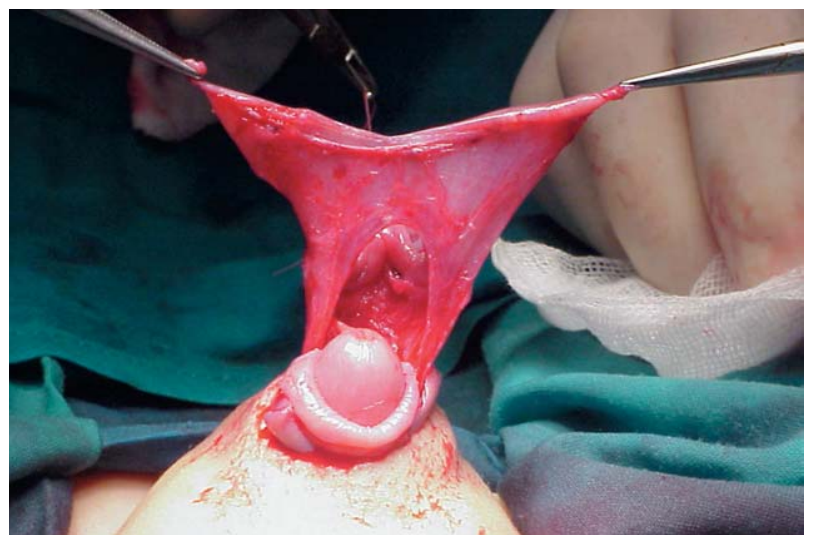

FIGURA $3 b$

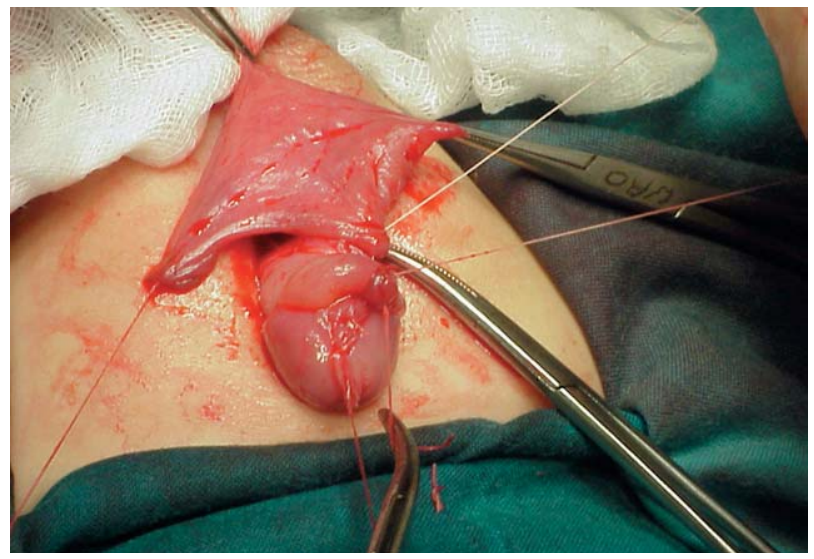

FIGURA 3c con la sutura de toda la corona prepucial con hilo reabsorbible de 5/0 y a la colocación de una sonda vesical de $8 \mathrm{Ch}$, que se retiró al día siguiente, con micción voluntaria totalmente normal (Figs. 4a, 4b y 4c).

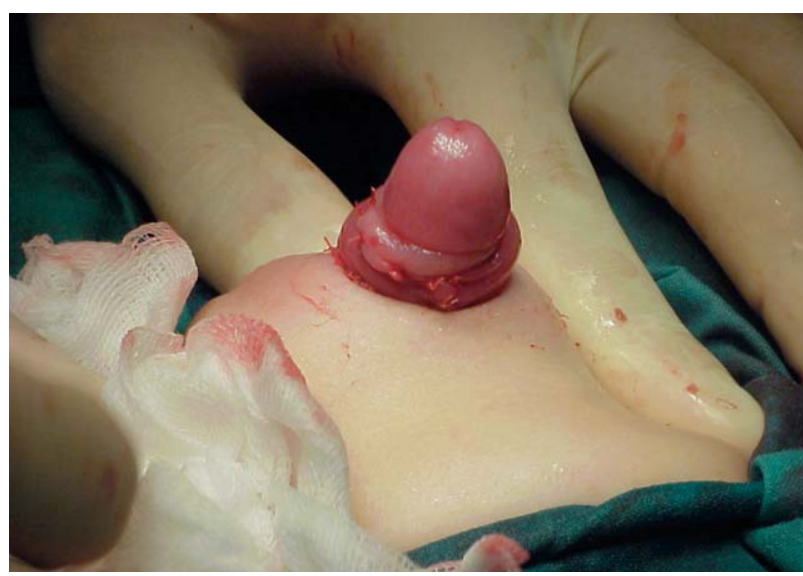

FIGURA $4 a$

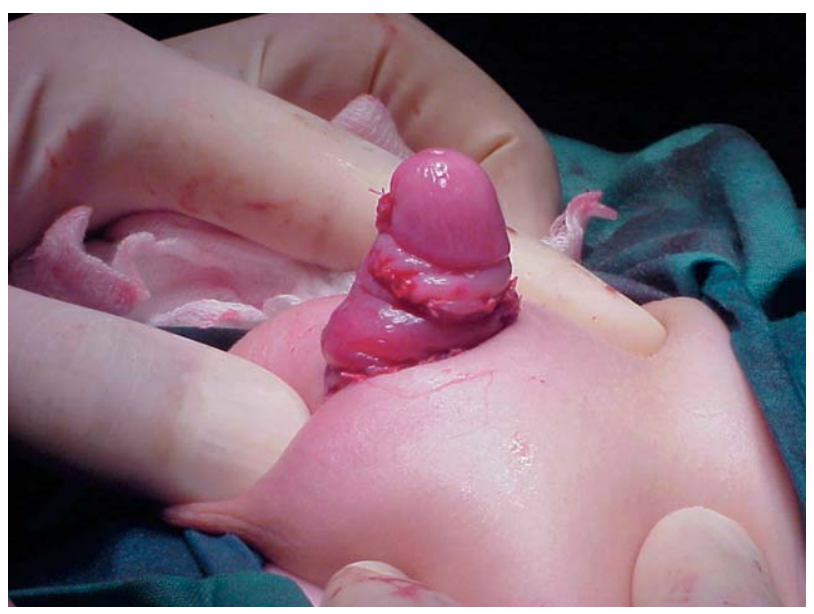

FIGURA 4b

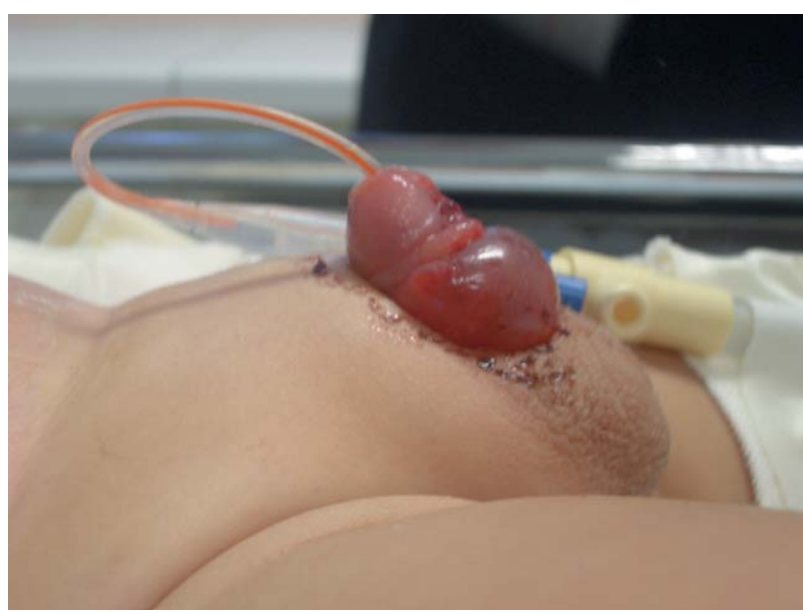

FIGURA $4 c$ 
Todos los pacientes se revisaron en la consulta externa al mes de la intervención quirúrgica, a los tres meses y al año de vida, sin objetivar complicación alguna.

Los resultados funcionales del procedimiento fueron evidentes, con una mejora importante en la micción del niño. El aspecto estético final del pene fue satisfactorio para todos y cada uno de los padres. De los 4 casos, tres de ellos quedaron con glande cubierto y uno de ellos con glande descubierto. (Figs. 5a y 5b).

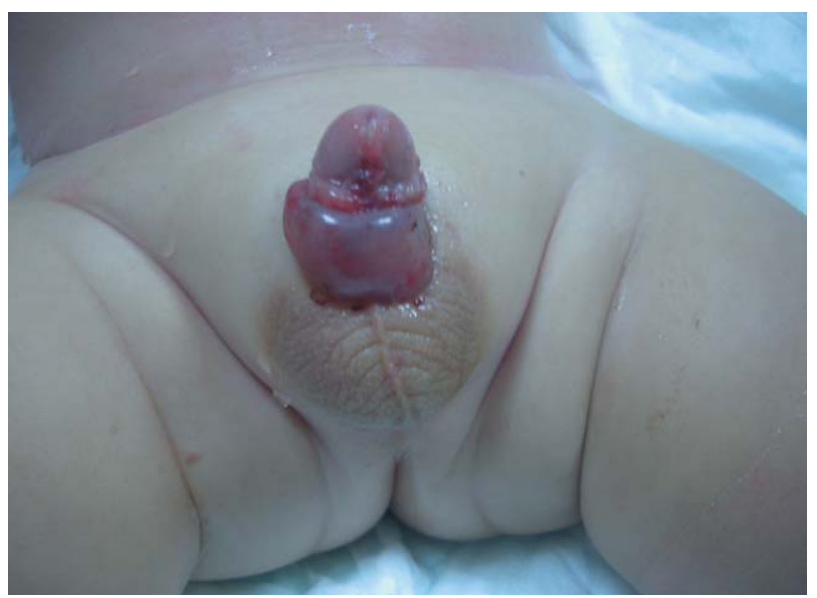

FIGURA $5 a$

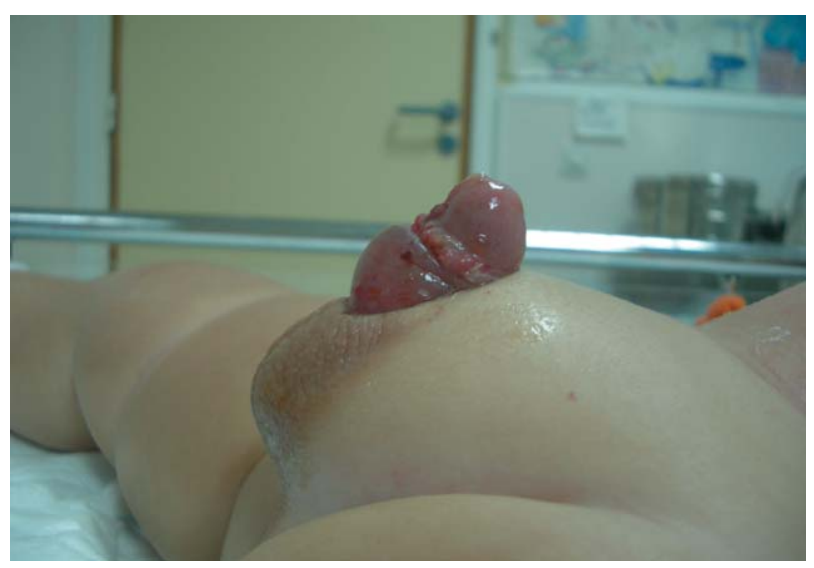

FIGURA $5 b$

\section{DISCUSIÓN}

El MC puede ser confundido con diversas y diferentes patologías como el pene enterrado o, más frecuentemente, con la fimosis ${ }^{4}$. El pene oculto o enterrado es una entidad que ha sido bien descrita y clasificada en la literatura, caracterizado por una piel peneana deficiente en su zona ventral y dorsal con una movilidad anormal sobre el pene del niño y por una implantación alta del rafe medio ${ }^{5,6}$. Además el pene enterrado puede asociarse a malformaciones uretrales como el hipospadias, que en ningún caso se asocian al MC. El exceso y redundancia de la mucosa prepucial que caracteriza al MC no está presente el pene enterrado ${ }^{4}$. La fimosis es aquella situación en la que el prepucio no puede retraerse completamente tras el glande o ésta es muy dificultosa. El origen del anillo fimótico es desconocida ${ }^{7}$. Esta entidad puede ocasionar un falso megaprepucio secundario a la estenosis prepucial propia de la fimosis. La incapacidad para descubrir el glande en el MC obedece a un defecto congénito de la piel ventral del prepucio, que acompañado de una mucosa prepucial muy redundante, ausente en niños con fimosis, diferencian claramente ambas situaciones clínicas. De la misma manera, la resolución quirúrgica de una y otra patología deben diferenciarse.

El diagnóstico del MC es clínico, mediante la exploración física ${ }^{3}$. Generalmente cursa con una masa o reservorio de contenido urinario durante la micción en la parte ventral del pene ${ }^{9,10}$. En ocasiones este hallazgo puede confundirse con patología diverticular de la uretra anterior ${ }^{3}$. Nosotros, en la actualidad, pensamos que no son necesarias otras exploraciones complementarias más cruentas, como la CUMS, ya que en caso de existir algún divertículo en uretra anterior, se puede diagnosticar y tratar intraoperatoriamente.

Hay autores que consideran que el tratamiento del megaprepucio, ya sea congénito o adquirido, debe ser la circuncisión precoz ${ }^{8}$. Al igual que Summerton, nosotros consideramos que esta opción de tratamiento no es la adecuada en estos casos, porque la resección quirúrgica excesiva de la mucosa prepucial, aún siendo abundante en el MC, impediría reparar con la misma el defecto de piel ventral, en ocasiones muy manifiesto, acompañante a esta condición ${ }^{4}$. Como se ha descrito anteriormente, el tratamiento del MC exige utilizar parte de la mucosa redundante en forma de colgajo pediculado, transponiendo el mismo a la cara ventral del pene, para con ello dejar lo más estéticamente posible el pene del niño.

Podemos concluir que el MC es una entidad poco frecuente $\mathrm{y}$ poco conocida. Teniendo en cuenta que es una condición que puede ser con- 
fundida con otras patologias, su sencillo tratamiento quirúrgico y su potencial capacidad para generar complicaciones en el tracto urinario del niño (obstrucción infravesical o ITUs), consideramos necesario reconocerla, diagnosticarla y tratarla adecuadamente.

\section{REFERENCIAS}

1. O`Brien A, Shapiro AMJ, Frank JD. Phimosis or congenital megaprepuce?. BJU. 1994;73(6):719-720.

2. Gomez J, Puyol M, Menendez V. Secondary Megaprepuce. BJU. 1996;78(6):959-960.

3. Serrano Durba A, Dominguez Hinarejos C, Reig Ruiz C. Secondary congenital megaprepuce. Scand J Urol Nephrol 1998;32(5):360-361.

4. Summerton DJ, McNally J, Denny AJ, Malone PS. Congenital megaprepuce: an emerging condition - how to recognize and treat it. BJU. 2000;86(4):519-522.

5. Maizels M, Zaontz M, Donovan J, Bushnick PN, Firlit CF Surgical correction of the buried penis:description of a classification system and a technique to correct the disorder. $\mathrm{J}$ Urol. 1986;136(1 Pt 2):268-271.
6. Wollin M, Duffy PG, Malone PS, Ransley PG. Buried penis: a novel approach. BJU. 1990;65(1):97-100.

7. Garat JM, Gosálbez R. Urologia Pediátrica. Ediciones Salvat S.A. 1987

8. Powis MR, Capps S. Prepucial intussusception or acquired megaprepuce. Paediatr Surg Int. 1998;13(2-3):158-159.

9. Bölükbasi A. Phimosis or congenital megaprepuce?. (Letter). BJU. 1995;76(1): 145-146.

10. Gwinn JL, Lee FA, Haber K. Radiological case of the month. Unusual presentation of phimosis. Am J Dis Child. 1974; 128(6):835-836.

Dr. FJ. Delgado Oliva

E-mail: frandelgol@hotmail.com

(Trabajo recibido el 24 de octubre 2005) 